\title{
Procesos Fenton como tratamiento complementario para la remoción de tensoactivos y coliformes de aguas residuales domésticas
}

\author{
Fenton processes as a complementary treatment for the removal \\ of surfactants and coliforms from municipal wastewater
}

\author{
David Naranjo-Tovar $^{1 *} \quad$ Leandro Morillo-Semanate ${ }^{1} \quad$ Jady Pérez $^{1}$ \\ William Villacis-Oñate $^{1} \quad$ Paul Vargas-Jentzsch $^{1} \quad$ Florinella Muñoz-Bisesti $^{1}$
}

Recibido 3 de abril de 2019, aceptado 29 de septiembre de 2020

Received: April 3, 2019 Accepted: September 29, 2020

\begin{abstract}
RESUMEN
La ineficiencia de los métodos convencionales para remover tensoactivos y patógenos de aguas residuales ha derivado en la necesidad de desarrollar tratamientos alternativos, como el proceso Fenton y sus variantes. En este estudio se aplicó un proceso Fenton convencional y modificado con ácido etilendiaminotetraacético (EDTA) como tratamiento complementario para aguas residuales rurales de Quito, Ecuador. Inicialmente, se establecieron las condiciones de concentración de peróxido de hidrógeno $\left(\mathrm{H}_{2} \mathrm{O}_{2}\right)$, la relación molar $\mathrm{Fe}^{2+}: \mathrm{H}_{2} \mathrm{O}_{2}$ y requerimiento de agitación para un proceso Fenton convencional. Estas condiciones se adaptaron a un proceso Fenton modificado con EDTA, en el cual se definieron las condiciones de relación molar $\mathrm{Fe}^{2+}$ :EDTA y de $\mathrm{pH}$. Las mejores condiciones establecidas fueron: concentración de $\mathrm{H}_{2} \mathrm{O}_{2}$ de $124,4 \mathrm{mM}$, agitación continua de $130 \mathrm{rpm}$, relación molar EDTA: $\mathrm{Fe}^{2+}: \mathrm{H}_{2} \mathrm{O}_{2}$ de 1:1:20 y pH de 6,41 (pH del efluente). Bajo estas condiciones, a partir de una muestra con concentración inicial de tensoactivos, coliformes fecales y DQO de $6,12 \mathrm{mg} / \mathrm{L}, 71000 \mathrm{UFC} / \mathrm{mL}$ y $980 \mathrm{mg} / \mathrm{L}$, respectivamente, se alcanzó inactivación total de coliformes fecales y una reducción de $97 \%$ para tensoactivos y 93\% para la demanda química de oxígeno a los 15 min de tratamiento. La concentración final de estos contaminantes cumplió con los estándares establecidos por la Agencia de Protección Ambiental (EPA). Este tratamiento podría, potencialmente, ser aplicado para complementar el tratamiento de aguas residuales que presenten problemas similares.
\end{abstract}

Palabras clave: Tensoactivos aniónicos, coliformes fecales, procesos de oxidación avanzada, Fenton modificado, EDTA.

\begin{abstract}
The inefficiency of conventional methods for removal surfactants and pathogens from wastewater has led to developing alternative treatments, such as the Fenton process and its variants. In this study, the conventional Fenton process and modified Fenton process with ethylenediaminetetraacetic acid (EDTA) were applied to reduce surfactants and fecal coliforms as a complementary treatment for wastewater in Quito, Ecuador. Initially, the conditions for hydrogen peroxide $\left(\mathrm{H}_{2} \mathrm{O}_{2}\right)$ concentration, molar ratio $\mathrm{Fe}^{2+}: \mathrm{H}_{2} \mathrm{O}_{2}$, and stirring were established for a conventional Fenton process; these conditions were after applied in a modified Fenton process with EDTA in which the conditions for the molar ratio $\mathrm{Fe}^{2+}$ :EDTA and $\mathrm{pH}$ were defined. The best experimental conditions were: $\mathrm{H}_{2} \mathrm{O}_{2}$ concentration of $124.4 \mathrm{mM}$, a continuous stirring

\footnotetext{
1 Escuela Politécnica Nacional. Facultad de Ingeniería Química y Agroindustria. Departamento de Ciencias Nucleares. Quito, Ecuador. E-mail: david.naranjo@epn.edu.ec; leandro.morillo@epn.edu.ec; jady.perez@epn.edu.ec; william.villacis@epn.edu.ec; paul.vargas@epn.edu.ec; florinella.munoz@epn.edu.ec
}

* Autor de correspondencia: david.naranjo@epn.edu.ec
\end{abstract}


of 130 rpm, molar ratio EDTA: $\mathrm{F}^{e 2}+: \mathrm{H}_{2} \mathrm{O}_{2}$ of 1:1:20, and $\mathrm{pH}$ of 6.41. With these conditions, from a sample with an initial concentration of surfactants, fecal coliforms, and COD of $6.12 \mathrm{mg} / \mathrm{L}, 71000 \mathrm{CFU} / \mathrm{mL}$, and $980 \mathrm{mg} / \mathrm{L}$, respectively, total inactivation of fecal coliforms was achieved, and it was obtained a reduction of $97 \%$ for surfactants and 93\% for chemical oxygen demand after 15 min of treatment. The final concentration of these pollutants met water quality standards, according to the Environmental Protection Agency (EPA). This treatment could be potentially applied to treat wastewater dealing with the same problem.

Keywords: Anionic surfactants, fecal coliforms, advanced oxidation process, modified Fenton, EDTA.

\section{INTRODUCCIÓN}

El vertido de aguas residuales sin tratar en los cuerpos de agua constituye una problemática ambiental de interés mundial. Se estima que el $80 \%$ de las aguas residuales de las ciudades se descargan directamente y sin tratar en los ríos, lagunas o en el mar [1]. Dentro de la extensa variedad de contaminantes presentes en estas aguas residuales se encuentran los compuestos refractarios y los patógenos [2].

Los contaminantes refractarios se caracterizan porque sus moléculas resisten los mecanismos de degradación asociados a los tratamientos por métodos convencionales. Entre las sustancias refractarias que típicamente se encuentran en las aguas residuales domésticas están los agentes tensoactivos, los cuales alteran significativamente las propiedades superficiales del agua [2] y favorecen la eutrofización [3]. Por otro lado, los patógenos presentes en las aguas residuales pueden provocar una variedad de enfermedades, incluidas la gastroenteritis, la tifoidea y el cólera [4]; debido a la complejidad asociada a la identificación individual de los patógenos presentes en las aguas residuales, su presencia es determinada a través de microorganismos indicadores como los coliformes fecales [2].

Tradicionalmente, los procesos aplicados para tratar las aguas residuales no han resultado del todo efectivos para disminuir la carga contaminante de tensoactivos, debido a su naturaleza refractaria, además, los tensoactivos en el agua forman espuma de gran estabilidad, que interfiere en el proceso convencional de aireación [5]. En cuanto a los procesos de desinfección de las aguas residuales, la cloración ha sido la técnica más ampliamente utilizada, sin embargo, durante dicho tratamiento se forman compuestos tóxicos, como trihalometanos, ácidos haloacéticos y subproductos de desinfección nitrogenados $[2,6]$.
En Quito (Ecuador), la planta de tratamiento de aguas residuales (PTAR) del barrio rural de Ubillús consiste en un proceso convencional de tratamiento en el cual el agua pasa a través de una fosa séptica hacia un filtro anaerobio de flujo ascendente (FAFA) $\mathrm{y}$, finalmente, el efluente se descarga directamente al río de la zona. Datos históricos de las características del efluente, proporcionados por la Empresa Pública Metropolitana de Agua Potable y Saneamiento del Distrito Metropolitano de Quito (EPMAPS), revelaron que la carga contaminante de tensoactivos, coliformes fecales y demanda química de oxígeno (DQO) suelen superar los límites establecidos por las normas de la Agencia de Protección Ambiental (EPA) [7, 8], tal como se muestra en la Tabla 1, lo que denota la ineficacia del tratamiento aplicado.

Ante las limitaciones de los procesos convencionales para el tratamiento de aguas residuales, se presentan como alternativa los procesos de oxidación avanzada (POAs) y, dentro de este grupo, los procesos Fenton. Los POAs se caracterizan por producir radicales hidroxilo $\left({ }^{\circ} \mathrm{OH}\right)$, los cuales reaccionan de forma no selectiva con los contaminantes orgánicos presentes en las aguas residuales [9]. En el proceso Fenton convencional, los 'OH son generados por la descomposición de peróxido de hidrógeno $\left(\mathrm{H}_{2} \mathrm{O}_{2}\right)$ en presencia de iones ferrosos $\left(\mathrm{Fe}^{2+}\right)$ y en medio ácido [9]. Se han planteado una serie de reacciones para explicar el complejo mecanismo del proceso Fenton, mismas que se muestran en las ecuaciones (1) a la (7) [9]:

$$
\begin{gathered}
\mathrm{Fe}^{2+}+\mathrm{H}_{2} \mathrm{O}_{2} \rightarrow \mathrm{F}^{3+}+\mathrm{OH}+\mathrm{HO}^{-} \\
\mathrm{Fe}^{3+}+\mathrm{H}_{2} \mathrm{O}_{2} \rightarrow \mathrm{Fe}^{2+}+\mathrm{HO}_{2}+\mathrm{H}^{+} \\
\mathrm{OH}+\mathrm{H}_{2} \mathrm{O}_{2} \rightarrow \mathrm{H}_{2} \mathrm{O}+\mathrm{H}_{2} \mathrm{O} \\
\mathrm{Fe}^{2+}+\mathrm{OH} \rightarrow \mathrm{Fe}^{3+}+\mathrm{HO}^{-}
\end{gathered}
$$


Tabla 1. Carga orgánica y presencia de coliformes fecales en el efluente de la PTAR de Ubillús (valores promedio de los años 2014-2015).

\begin{tabular}{|l|c|c|}
\hline \multicolumn{1}{|c|}{ Parámetro } & Valor histórico $^{\mathbf{1}}$ & Límite Máximo Permisible $^{\mathbf{2}}$ \\
\hline DQO (mg/L) & $961,72 \pm 74,20$ & 160,00 \\
\hline Tensoactivos (mg/L) & $8,18 \pm 1,51$ & 0,20 \\
\hline Coliformes fecales (UFC/mL) & $61455,55 \pm 1372,25$ & 10,00 \\
\hline
\end{tabular}

1 media \pm desviación estándar.

2 Límite Máximo Permisible para descarga de aguas residuales domésticas tratadas a cuerpos de agua según la EPA.

Fuente: EPMAPS

$$
\begin{gathered}
\mathrm{Fe}^{3+}+\mathrm{HO}_{2} \rightarrow \mathrm{Fe}^{2+}+\mathrm{O}_{2} \mathrm{H}^{+} \\
\mathrm{Fe}^{2+}+\mathrm{HO}_{2}+\mathrm{H}^{+} \rightarrow \mathrm{Fe}^{3+}+\mathrm{H}_{2} \mathrm{O}_{2} \\
\mathrm{HO}_{2}+\mathrm{HO}_{2} \rightarrow \mathrm{H}_{2} \mathrm{O}_{2}+\mathrm{O}_{2}
\end{gathered}
$$

Este proceso se ha utilizado para degradar contaminantes del agua como colorantes, organohalógenos, compuestos aromáticos policíclicos [9] y tensoactivos [10]; asimismo, se ha reducido la DQO y se ha inactivado E. coli, tanto en muestras sintéticas como en efluentes reales [11]. La necesidad de trabajar en condiciones ácidas ( $\mathrm{pH}$ aproximadamente 3,0 ) es fundamental, caso contrario se afecta negativamente a la producción efectiva de ${ }^{\circ} \mathrm{OH}$ y/o se provoca la precipitación del hierro en forma de oxihidróxidos [9]. La adición de un agente quelante del hierro permite modificar el proceso debido a la formación del complejo quelantemetal, que mantiene soluble al hierro en un rango más amplio de valores de $\mathrm{pH}$ [12]. Se han utilizado varios ácidos carboxílicos como agentes quelantes, sin embargo, uno de los más estudiados ha sido el ácido etilendiaminotetraacético (EDTA), el cual destaca por su efectividad para quelar el hierro y, por tanto, este complejo EDTA-Fe ${ }^{2+}$ fue usado como catalizador en el proceso Fenton modificado [13, 14]. Se ha comprobado la eficacia del uso del EDTA como quelante del hierro en procesos Fenton para la remoción efectiva de compuestos como la goma guar y la rodamina $\mathrm{B}$, y se verificó la posibilidad de variar el $\mathrm{pH}$ del medio reaccionante $[15,16]$.

En respuesta a los antecedentes presentados, se estudió la reducción de tensoactivos y coliformes fecales mediante los procesos Fenton convencional y modificado con EDTA, como tratamiento complementario para las aguas residuales rurales de la PTAR de Ubillús.

\section{METODOLOGÍA}

\section{Caracterización química y microbiológica de las aguas residuales pretratadas descargadas a un cauce de agua}

Se llevó a cabo una etapa de muestreo aleatorio en la que se tomaron un total de nueve muestras simples del efluente de la PTAR por un período de ocho semanas. El volumen de cada muestra simple fue de $1 \mathrm{~L}$ y se tomó a la salida del FAFA. Las muestras se preservaron de acuerdo con la metodología APHA 1060 y APHA 9060 [17]. En cada muestra simple se analizó la concentración de tensoactivos, coliformes fecales y el valor de $\mathrm{pH}$.

La cuantificación de tensoactivos se llevó a cabo de acuerdo con la metodología descrita en APHA 5540 C [17]. Se utilizó un espectrofotómetro UV-Vis Thermo Scientific Genesys 20. El recuento de coliformes fecales se realizó con el uso de Petrilm ${ }^{\mathrm{TM}} 3 \mathrm{M}$, de acuerdo con la metodología NMKL 147. Se utilizó una cámara de flujo laminar Labconco 36000, una incubadora Memmert B 30 y un autoclave All American 25 X [18]. La medición del valor de pH se llevó a cabo de acuerdo con el método APHA 4500-H +B. Se utilizó el medidor de pH Milwaukee Mi 151 [17].

Los análisis se llevaron a cabo por duplicado. Con los valores obtenidos de los análisis de las muestras simples se construyeron cartas de control, se determinó la variación estadística de los parámetros de estudio y, de acuerdo con ello, se estableció un plan de muestreo compuesto. El volumen de la muestra compuesta fue de $16 \mathrm{~L}$ y estuvo constituida 
por cuatro submuestras de $4 \mathrm{~L}$ que se tomaron cada $2 \mathrm{~h}$ a la salida del FAFA.

Determinación de la concentración de $\mathrm{H}_{2} \mathrm{O}_{2}$ y requerimiento de agitación en un proceso Fenton convencional que permitiera la mayor reducción de tensoactivos y coliformes

Se utilizó sulfato ferroso heptahidratado $\left(\mathrm{FeSO}_{4} \cdot 7 \mathrm{H}_{2} \mathrm{O}\right.$ $>99 \%$ p/p), peróxido de hidrógeno $\left(\mathrm{H}_{2} \mathrm{O}_{2}, 30 \%\right.$ $\mathrm{p} / \mathrm{p})$ y solución de ácido sulfúrico $\left(\mathrm{H}_{2} \mathrm{SO}_{4}\right) 1 \mathrm{~N}$.

Para llevar a cabo el tratamiento mediante un proceso Fenton convencional, se acondicionó el $\mathrm{pH}$ de la muestra del efluente mediante la adición de solución de $\mathrm{H}_{2} \mathrm{SO}_{4} 1 \mathrm{~N}$. Se añadió la cantidad necesaria de $\mathrm{FeSO}_{4} \cdot 7 \mathrm{H}_{2} \mathrm{O}$ y se homogeneizó la mezcla. Se añadió el volumen requerido de $\mathrm{H}_{2} \mathrm{O}_{2}$ para iniciar la reacción. El reactor estuvo constituido por un vaso de precipitación de $1000 \mathrm{~mL}$ con un agitador de paleta del equipo Phipps \& Bird s.7790900 de regulación automática.

En esta etapa se aplicó el tratamiento Fenton convencional en la muestra compuesta y se trabajó con un diseño experimental $3 \times 2$. Las variables de diseño fueron la relación en masa tensoactivos: $\mathrm{H}_{2} \mathrm{O}_{2}$ y la agitación. La relación en masa tensoactivos: $\mathrm{H}_{2} \mathrm{O}_{2}$ se varió en tres niveles: 1:450, 1:350 y 1:250, los mismos que fueron definidos a partir de ensayos preliminares. La agitación se varió en dos niveles: 130 rpm y sin agitación continua. Las variables de respuesta fueron las concentraciones de tensoactivos y de coliformes fecales. El volumen experimental fue de $100 \mathrm{~mL}$ y se realizaron ensayos por duplicado [19]. Las demás condiciones del tratamiento fueron relación molar $\mathrm{Fe}^{2+}: \mathrm{H}_{2} \mathrm{O}_{2}$ igual a 1:5, valor de $\mathrm{pH}$ 2,8; temperatura ambiente (entre 17 y $19^{\circ} \mathrm{C}$ ), sistema batch, durante $60 \min [10,11]$.

La concentración de $\mathrm{H}_{2} \mathrm{O}_{2}$ a utilizarse en los ensayos posteriores se estableció de acuerdo con la relación en masa tensoactivos: $\mathrm{H}_{2} \mathrm{O}_{2}$ que permitió la mayor remoción de los contaminantes en estudio y se recalculó para la mayor concentración histórica de tensoactivos obtenida en la etapa de caracterización.

Establecimiento de la relación molar $\mathrm{Fe}^{2+}: \mathrm{H}_{2} \mathrm{O}_{2}$ del proceso Fenton convencional que permitiera la mayor reducción de los contaminantes en estudio Se complementó el estudio del tratamiento Fenton convencional mediante un diseño experimental unifactorial. La variable de diseño fue la relación molar $\mathrm{Fe}^{2+}: \mathrm{H}_{2} \mathrm{O}_{2}$, la cual se varió en tres niveles: 1:5, $1: 10$ y $1: 20[10,11]$. Las variables de respuesta fueron las concentraciones de tensoactivos y coliformes fecales. El volumen experimental fue de $600 \mathrm{~mL}$ y los ensayos se realizaron por duplicado. Las demás condiciones del tratamiento fueron valor de $\mathrm{pH} 2,8$; temperatura ambiente (entre 17 y $19^{\circ} \mathrm{C}$ ) y sistema batch. La concentración de $\mathrm{H}_{2} \mathrm{O}_{2}$ y el requerimiento de agitación con los que se trabajaron en esta etapa fueron previamente definidos. Se realizaron análisis de la reducción de los contaminantes a los 15, 30, $60,90,120$ y $180 \min [10,11]$.

\section{Determinación de la relación molar $\mathrm{Fe}^{2+}$ :EDTA y valor de $\mathbf{p H}$ del proceso Fenton modificado que permitiera la mayor reducción de los contaminantes en estudio}

Además de los reactivos utilizados en las etapas anteriores se utilizó sal disódica dihidratada de EDTA $\left(\mathrm{C}_{10} \mathrm{H}_{14} \mathrm{~N}_{2} \mathrm{Na}_{2} \mathrm{O}_{8} \cdot 2 \mathrm{H}_{2} \mathrm{O},>99 \% \mathrm{p} / \mathrm{p}\right)$ y solución de hidróxido de sodio $(\mathrm{NaOH}) 1 \mathrm{~N}$.

Para llevar a cabo el tratamiento mediante un proceso Fenton modificado con EDTA, se preparó inicialmente la solución concentrada del complejo EDTA- $\mathrm{Fe}^{2+}$, con las relaciones molares requeridas para cada ensayo. Las condiciones de preparación del complejo fueron temperatura ambiente (entre 17 y $19{ }^{\circ} \mathrm{C}$ ), valor de $\mathrm{pH} 2,8$ y agitación continua durante $30 \mathrm{~min}$. Finalizado este proceso, se neutralizó la solución con la adición de solución de $\mathrm{NaOH}$ $1 \mathrm{~N}$ [13]. Se añadió el complejo a la muestra del efluente y se reguló el $\mathrm{pH}$ mediante la adición de $\mathrm{H}_{2} \mathrm{SO}_{4} 1 \mathrm{~N}$ o de $\mathrm{NaOH} 1 \mathrm{~N}$, según lo requerido en cada caso. Finalmente, se añadió el volumen necesario de $\mathrm{H}_{2} \mathrm{O}_{2}$ para iniciar la reacción.

Se aplicó el tratamiento Fenton modificado con EDTA en la muestra compuesta y se trabajó con un diseño experimental $4 \times 2$. Las variables de diseño fueron la relación molar $\mathrm{Fe}^{2+}$ :EDTA y el valor de pH. La relación molar $\mathrm{Fe}^{2+}$ :EDTA se varió en dos niveles: 1:1 y 4:5 [12]. El valor del $\mathrm{pH}$ se varió en cuatro niveles que fueron: 4,50; 6,00; $8,00 \mathrm{y}$ el pH propio del efluente, es decir, sin acondicionamiento. Las variables de respuesta fueron las concentraciones de tensoactivos y coliformes fecales. El volumen experimental fue de $600 \mathrm{~mL}$ y los ensayos se realizaron por duplicado. Las demás condiciones del tratamiento fueron temperatura ambiente (entre $17 \mathrm{y}$ 
$19^{\circ} \mathrm{C}$ ) y sistema batch. La concentración de $\mathrm{H}_{2} \mathrm{O}_{2}$, el requerimiento de agitación y la relación molar $\mathrm{Fe}^{2+}: \mathrm{H}_{2} \mathrm{O}_{2}$ con los que se trabajaron en esta etapa se definieron en las etapas previas. Se realizaron análisis de la reducción de los contaminantes a los $15,30,60,90,120$ y $180 \min [20]$.

Evaluación de la remoción global de los contaminantes en el efluente, a través de la DQO, bajo las mejores condiciones de Fenton modificado con EDTA

Se llevó a cabo un ensayo bajo las condiciones de Fenton modificado con EDTA definidas en la sección anterior.

Se aplicó el tratamiento en la muestra compuesta y se realizaron mediciones de la concentración de tensoactivos, coliformes fecales y DQO, antes y después del tratamiento mencionado anteriormente. El ensayo se realizó por triplicado. El análisis de la DQO se realizó de acuerdo con la metodología APHA 5220 D. Se utilizó un reactor DQO HACH y un espectrofotómetro HACH DR 2800 [17]. El análisis estadístico de todos los datos se efectuó en el software STATGRAPHICS Centurion XVI versión 16.1.03, mediante análisis de la varianza, diagramas de Pareto y gráficas de medias.

\section{RESULTADOS}

\section{Caracterización química y microbiológica de las aguas residuales pretratadas descargadas a un cauce de agua}

En la Figura 1 se presentan las cartas de control construidas a partir de los resultados obtenidos en la etapa de muestreo aleatorio del efluente de la PTAR. Se aprecia que para todos los parámetros caracterizados: concentración de tensoactivos expresados como sustancias activas al azul de metileno (SAAM), coliformes fecales (EC) y pH, los valores reportados se encontraron entre los límites de control superior (LCS) e inferior (LCI), e inclusive entre los límites de alarma superior (LAS) e inferior (LAI). Este resultado implicó que los parámetros en estudio se mantuvieron bajo control estadístico a lo largo del tiempo y, por consiguiente, se estableció que el muestreo compuesto podría realizarse en cualquier día de operación normal de la planta [17]. Todos los valores históricos de concentración de tensoactivos y coliformes fecales sobrepasaron los límites máximos permisibles indicados por la norma de descarga a un cuerpo de agua dulce de la EPA, que establece una concentración máxima de $0,20 \mathrm{mg} / \mathrm{L}$ para tensoactivos y $1000 \mathrm{UFC} / 100 \mathrm{~mL}$ para coliformes fecales [7]. Esto reafirmó la necesidad de desarrollar un proceso que complementase el tratamiento biológico aplicado en la PTAR.

Por otro lado, se observó que los valores históricos de $\mathrm{pH}$ se encontraron dentro del rango indicado por la norma anteriormente mencionada, que establece los valores límite de 6,5 y 9,0, con un límite inferior de hasta 6,0, siempre que la concentración de $\mathrm{CO}_{2}$ libre no supere las 100 ppm [8]. La máxima concentración de tensoactivos fue de $9,40 \mathrm{mg} / \mathrm{L}$, a partir de este valor se definió la concentración de $\mathrm{H}_{2} \mathrm{O}_{2}$ que se utilizó en los ensayos subsiguientes.

Determinación de la concentración de $\mathrm{H}_{2} \mathrm{O}_{2}$ y requerimiento de agitación en un proceso Fenton convencional que permitiera la mayor reducción de tensoactivos y coliformes

En la Figura 2 se presentan los resultados de la variación del porcentaje de remoción de los contaminantes en estudio en la muestra compuesta, tratada mediante un proceso Fenton convencional. La carga inicial de contaminantes en la muestra fue de $5,20 \mathrm{mg} / \mathrm{L}$ para tensoactivos y $66000 \mathrm{UFC} / \mathrm{mL}$ para coliformes fecales.

Se observó que, para la reducción de tensoactivos, el mayor porcentaje de remoción fue cercano al $73 \%$ respecto a la concentración inicial, este resultado se alcanzó al aplicar el tratamiento con agitación continua ( $130 \mathrm{rpm}$ ) y la mayor concentración de $\mathrm{H}_{2} \mathrm{O}_{2}$ (relación en masa tensoactivos: $\mathrm{H}_{2} \mathrm{O}_{2}$ de 1:450). El análisis estadístico de los resultados reveló que tanto la agitación como la concentración de $\mathrm{H}_{2} \mathrm{O}_{2}$ presentaron un efecto significativo durante el tratamiento.

La importancia de la agitación durante el proceso radicó en que este parámetro aumenta el contacto entre las especies reactantes del medio, y por tanto incrementa la probabilidad de que ocurran las reacciones; asimismo, la agitación permite la absorción de oxígeno en el efluente, este gas actúa como un agente oxidante suplementario e incrementa la velocidad de mineralización de los contaminantes $[9,21]$.

No obstante, el efecto de la absorción de oxígeno puede considerarse despreciable, debido a las 

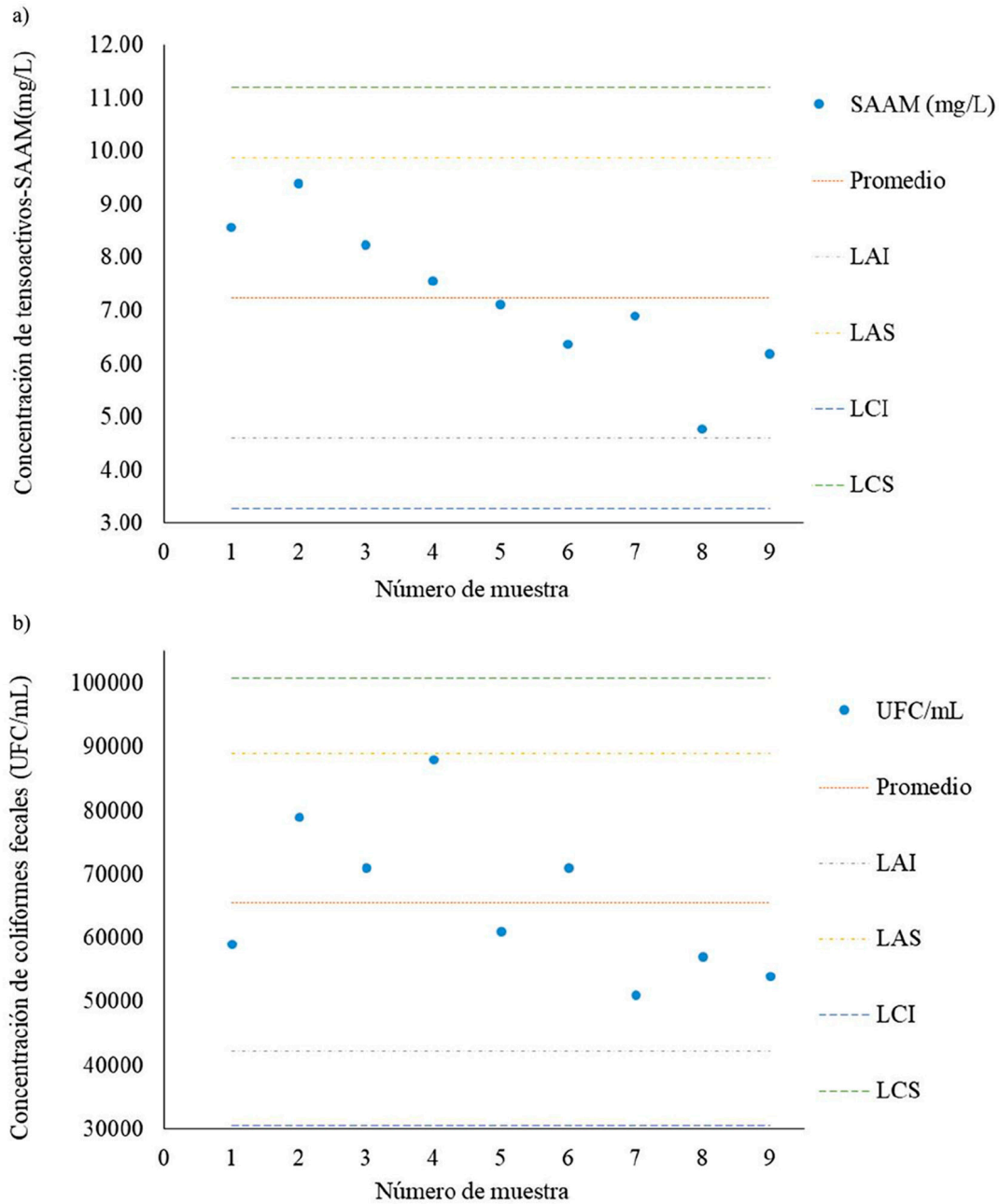

c)

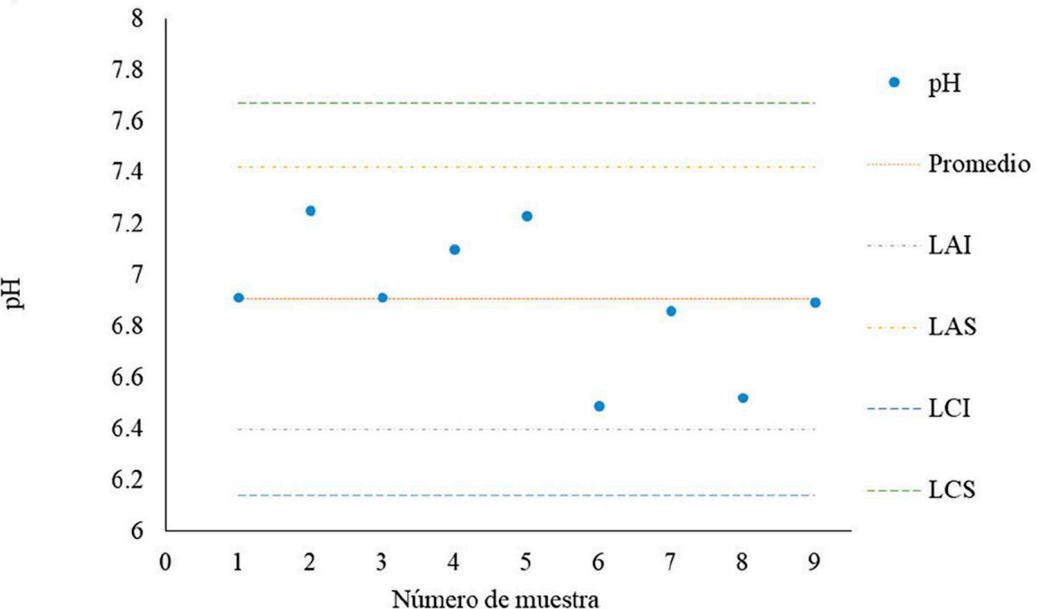

Figura 1. Cartas de control estadístico de los parámetros: (a) concentración de tensoactivos (SAAM) (mg/L), (b) concentración de coliformes fecales (EC) (UFC/mL) y (c) pH del efluente de la PTAR del barrio Ubillús, medidos por un período de 8 semanas. 

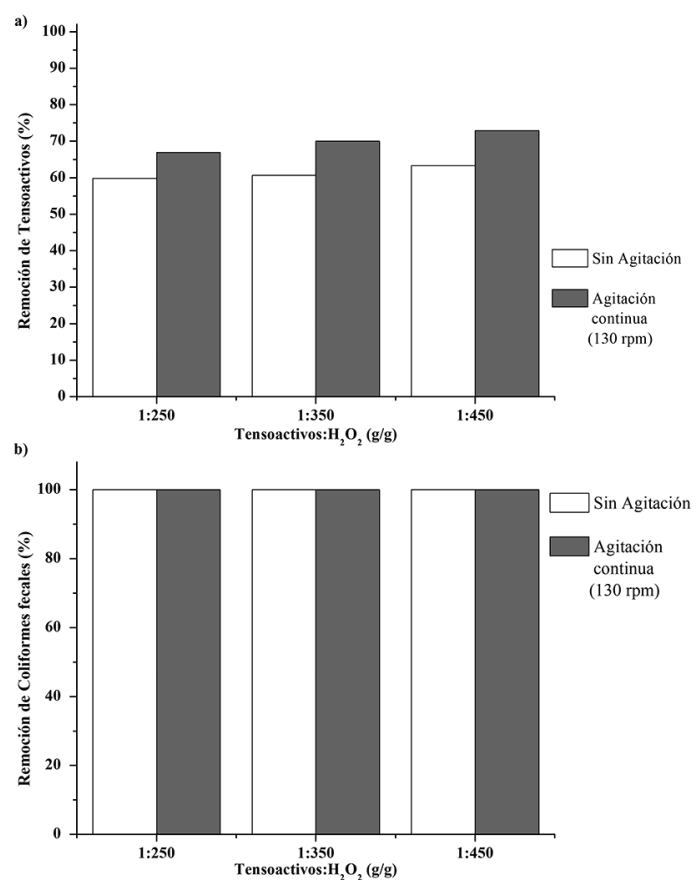

Figura 2. Porcentaje de remoción de a) tensoactivos (SAAM) y b) coliformes fecales (EC) en el efluente de la PTAR de Ubillús, tratado mediante Fenton convencional con diferentes relaciones en masa tensoactivos: $\mathrm{H}_{2} \mathrm{O}_{2}$ e intensidades de agitación $\left(\mathrm{Fe}^{2+}: \mathrm{H}_{2} \mathrm{O}_{2}=1: 5 ; \mathrm{pH}=2,8 ; \mathrm{t}\right.$ $=60 \mathrm{~min} ;[\mathrm{SAAM}]_{0}=5,20 \mathrm{mg} / \mathrm{L} ;[\mathrm{EC}]_{0}$ $=66000 \mathrm{UFC} / \mathrm{mL}$ )

condiciones experimentales bajo las que se realizaron los ensayos.

Por otro lado, la concentración de $\mathrm{H}_{2} \mathrm{O}_{2}$ afectó directamente a la eficacia del proceso, dado que es el reactivo a partir del cual se producen los ' $\mathrm{OH}$ [9, $22,23]$. De acuerdo con los resultados obtenidos, se observó una marcada tendencia de aumentar la remoción de tensoactivos con el aumento de la concentración de $\mathrm{H}_{2} \mathrm{O}_{2}$, por tanto, se consideró que en el rango de concentración de $\mathrm{H}_{2} \mathrm{O}_{2}$ utilizado en esta etapa, este reactivo no se comportó como scavenger de los ' $\mathrm{OH}$ y, en consecuencia, se estableció que, en los ensayos subsiguientes, se trabajaría con la mayor concentración de $\mathrm{H}_{2} \mathrm{O}_{2}$ definida por la relación en masa tensoactivos: $\mathrm{H}_{2} \mathrm{O}_{2}$ de 1:450.
Se alcanzó inactivación total de coliformes fecales en todos los experimentos realizados en esta etapa. Uno de los modelos, mayoritariamente aceptados, por el que se describe el mecanismo de muerte celular durante la reacción Fenton, es aquel en el cual los ${ }^{\circ} \mathrm{OH}$ promueven la oxidación de los fosfolípidos que componen la membrana celular, lo que desencadena en lisis celular [24].

La eficacia del proceso Fenton para la desinfección de efluentes se ha comprobado en varias investigaciones. Se ha reportado inactivación total de $E$. coli, en menos de 1 min de haber tratado aguas residuales urbanas mediante un proceso Fenton convencional a partir de una carga inicial de $38000 \mathrm{UFC} / \mathrm{mL}$ y con una concentración de $\mathrm{H}_{2} \mathrm{O}_{2}$ de 114,9 mM [11].

Por otro lado, han alcanzado tres reducciones logarítmicas de E. coli a partir de una carga inicial de $70000 \mathrm{UFC} / \mathrm{mL}$ al usar una concentración de $\mathrm{H}_{2} \mathrm{O}_{2}$ de $1,5 \mathrm{mM}$ para tratar aguas residuales municipales [25].

Al comparar los resultados de las investigaciones mencionadas con los resultados obtenidos en los experimentos llevados a cabo en esta etapa (en los que se trabajó en un rango de concentraciones de $\mathrm{H}_{2} \mathrm{O}_{2}$ entre 38,2 a $68,8 \mathrm{mM}$ y con una carga inicial de coliformes fecales de $66000 \mathrm{UFC} / \mathrm{mL}$ ), se apreció que el tratamiento de desinfección mediante un proceso Fenton depende, de manera general, de la carga inicial de microorganismos y, principalmente, de la concentración de $\mathrm{H}_{2} \mathrm{O}_{2}$ utilizada, sin descartar las posibles interferencias provocadas por los demás contaminantes presentes en los efluentes tratados.

Los resultados obtenidos en esta etapa permitieron justificar el hecho de haber definido la concentración de $\mathrm{H}_{2} \mathrm{O}_{2}$ con base en la carga contaminante de tensoactivos y no de coliformes fecales, pues la resistencia de estos últimos frente a los mecanismos de degradación durante el proceso Fenton fue relativamente menor que aquella presentada por los tensoactivos.

De este modo, con los resultados obtenidos de esta etapa y de la etapa de caracterización, se determinó que los experimentos subsiguientes se llevarían a cabo con agitación continua (130 rpm) y con la concentración de $\mathrm{H}_{2} \mathrm{O}_{2}$ recalculada para la mayor concentración histórica de tensoactivos $9,40 \mathrm{mg} / \mathrm{L}$, con la relación en masa tensoactivos: $\mathrm{H}_{2} \mathrm{O}_{2}$ de 1:450, que resultó 124,4 mM. 
La razón por la cual se usó la relación en masa tensoactivos: $\mathrm{H}_{2} \mathrm{O}_{2}$, se debió al hecho de que se trabajó con un efluente real en el cual era muy poco probable que se tuviera un solo tipo de tensoactivo, este aspecto dificulta la determinación de la masa molar correspondiente a dicho contaminante. Empero, se puede realizar una aproximación teórica al tomar la masa molar del tensoactivo utilizado como referencia en la metodología de caracterización estandarizada, dicho tensoactivo es el sulfonato de alquilbenceno lineal (LAS) cuya masa molar promedio es $342,4 \mathrm{~g} / \mathrm{mol}$ [17].

Al realizar los cálculos estequiométricos correspondientes, se obtuvo que la relación molar equivalente a la relación en masa tensoactivos: $\mathrm{H}_{2} \mathrm{O}_{2}$ de 1:450 fue de 1:4525. Esta relación molar, a su vez, puede compararse con el requerimiento estequiométrico de $\mathrm{H}_{2} \mathrm{O}_{2}$ necesario para oxidar completamente al LAS. Se conoce que $1 \mathrm{mg} / \mathrm{L}$ de LAS equivale a $2,7 \mathrm{mg} / \mathrm{L}$ de DQO [26], además, si se considera que el $\mathrm{H}_{2} \mathrm{O}_{2}$ se descompone de acuerdo con la ecuación (8), el requerimiento estequiómetrico expresado como relación molar tensoactivos: $\mathrm{H}_{2} \mathrm{O}_{2}$ resultante es 1:58.

$$
2 \mathrm{H}_{2} \mathrm{O}_{2} \rightarrow 2 \mathrm{H}_{2} \mathrm{O}+\mathrm{O}_{2}
$$

En consecuencia, la relación molar tensoactivos: $\mathrm{H}_{2} \mathrm{O}_{2}$ con la que se trabajó fue aproximadamente 78 veces mayor al requerimiento estequiométrico. Este notable exceso tomaría relevancia si se hubiera trabajado con muestras sintéticas, pero al tratarse de un efluente real el $\mathrm{H}_{2} \mathrm{O}_{2}$ se utilizó para generar radicales que reaccionaran no solo con los tensoactivos, sino también con otros contaminantes presentes en el agua.

Establecimiento de la relación molar $\mathrm{Fe}^{2+}: \mathrm{H}_{2} \mathrm{O}_{2}$ del proceso Fenton convencional que permitiera la mayor reducción de los contaminantes en estudio Los resultados presentados en la Figura 3 corresponden a la variación en el tiempo del porcentaje remanente de tensoactivos en la muestra compuesta, tratada mediante un proceso Fenton convencional.

En el experimento llevado a cabo en la etapa anterior, en el que se trabajó con la relación molar $\mathrm{Fe}^{2+}: \mathrm{H}_{2} \mathrm{O}_{2}$ de 1:5 y una concentración de $\mathrm{H}_{2} \mathrm{O}_{2}$ de $68,8 \mathrm{mM}$ el porcentaje remanente de tensoactivos

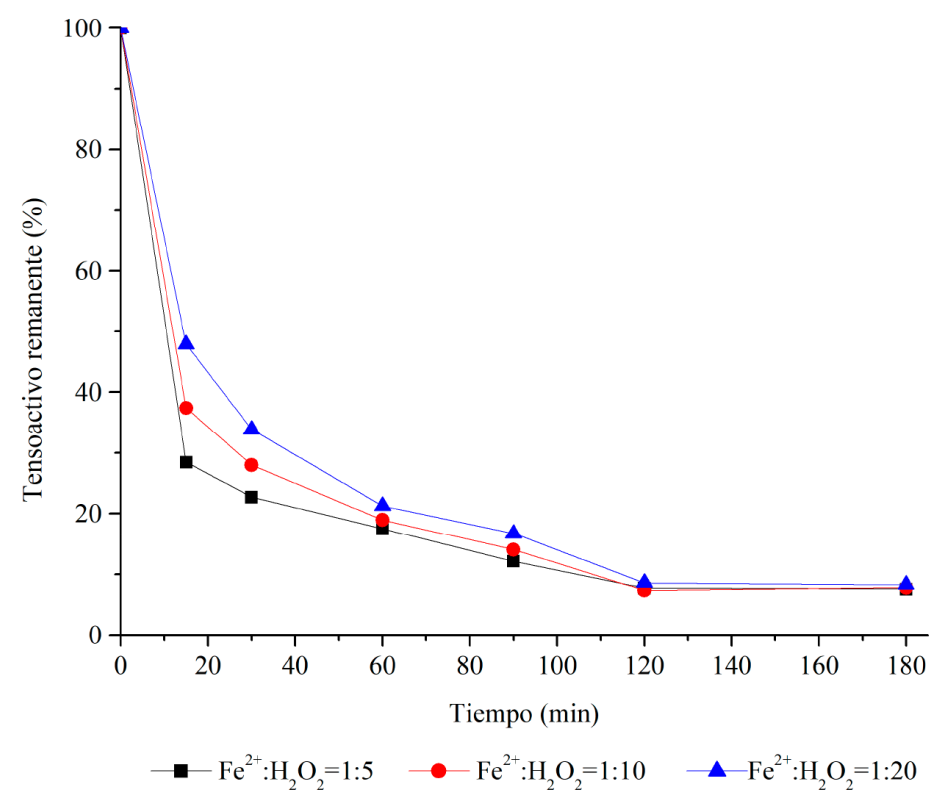

Figura 3. Variación del porcentaje remanente de tensoactivos (SAAM) en el efluente de la PTAR de Ubillús, tratado mediante Fenton convencional con diferentes relaciones molares $\mathrm{Fe}^{2+}: \mathrm{H}_{2} \mathrm{O}_{2}\left(\left[\mathrm{H}_{2} \mathrm{O}_{2}\right]=124,4 \mathrm{mM} ; \mathrm{pH}=2,8\right.$; agitación = $\left.130 \mathrm{rpm}[\mathrm{SAAM}]_{0}=5,20 \mathrm{mg} / \mathrm{L}\right)$. 
fue de $27 \%$ a los 60 min de tratamiento, mientras que en el experimento llevado a cabo en la presente etapa, al utilizar la misma relación molar $\mathrm{Fe}^{2+}: \mathrm{H}_{2} \mathrm{O}_{2}$, para el mismo tiempo de tratamiento, pero con una concentración de $\mathrm{H}_{2} \mathrm{O}_{2}$ de $124,4 \mathrm{mM}$, el porcentaje remanente de tensoactivos resultó aproximadamente $17 \%$.

Al comparar estos resultados, se pudo corroborar lo establecido anteriormente en cuanto a la influencia de la concentración de $\mathrm{H}_{2} \mathrm{O}_{2}$ en la eficacia del proceso Fenton, pues el uso de una mayor concentración de este reactivo incrementó el porcentaje de remoción de tensoactivos. En los resultados presentados en la Figura 3, se observa que se obtuvo un mayor porcentaje de remoción de tensoactivos en menos tiempo cuando se utilizó una mayor proporción de hierro. De este modo se podría considerar que la cantidad de hierro presente en el medio influyó directamente en la cinética del proceso $[9,22,23]$. No obstante, se observó que el efecto de la proporción de hierro sobre la cinética del proceso fue más notorio durante los 30 primeros minutos de tratamiento. Este comportamiento se debió, probablemente, a que la velocidad de reacción con la que el $\mathrm{Fe}^{2+}$ pasa a $\mathrm{Fe}^{3+}$ para formar ${ }^{\circ} \mathrm{OH}$, de acuerdo con la ecuación (1), es mucho mayor que la velocidad con la que las especies de $\mathrm{Fe}^{3+}$ se regeneran a $\mathrm{Fe}^{2+}$, cuya reacción se describe mediante la ecuación (2). Estas velocidades que corresponden aproximadamente a $70 \mathrm{M}^{-1} \mathrm{~s}^{-1}$ para el consumo del $\mathrm{Fe}^{2+}$, y entre $0,001 \mathrm{y}$ $0,010 \mathrm{M}^{-1} \mathrm{~s}^{-1}$ para su regeneración a partir de $\mathrm{Fe}^{3+}[9$, 27], permitieron explicar el hecho de que el proceso Fenton convencional ocurrió más rápidamente al inicio y conforme avanzó el tiempo la velocidad de reacción disminuyó hasta la finalización del tratamiento.

El análisis de los resultados de esta etapa permitió determinar que tanto la cantidad de hierro utilizada como el tiempo de tratamiento tuvieron un efecto estadísticamente significativo durante el proceso. Se pudo notar que, en todos los casos, la reacción prácticamente se detuvo alrededor de los $120 \mathrm{~min}$. Por tal razón, se decidió realizar un análisis estadístico con los porcentajes de remoción alcanzados al terminar la reacción. Los resultados de este análisis permitieron determinar que no existieron diferencias estadísticamente significativas en los porcentajes de remoción de tensoactivos entre los tres experimentos llevados a cabo con distintas relaciones molares
$\mathrm{Fe}^{2+}: \mathrm{H}_{2} \mathrm{O}_{2}$. En varias investigaciones en las que se ha utilizado Fenton convencional para el tratamiento de contaminantes en el agua, se ha procurado trabajar, en lo posible, con la mínima cantidad de hierro definida experimentalmente. La razón de esto radica en el hecho de que los iones ferrosos pueden actuar como scavengers de los ${ }^{\circ} \mathrm{OH}$, de acuerdo con la ecuación (4); del mismo modo, una mayor cantidad de hierro aumenta la probabilidad de que precipiten compuestos de este metal, como consecuencia de que se supere la solubilidad en agua de las especies de $\mathrm{Fe}^{3+}$ que se forman en el transcurso de la reacción Fenton [25, 28].

Como consecuencia de los resultados obtenidos y de su respectivo análisis, se estableció que en los experimentos subsiguientes se trabajaría con la menor cantidad de hierro utilizada en esta etapa, dada por la relación molar $\mathrm{Fe}^{2+}: \mathrm{H}_{2} \mathrm{O}_{2}$ de 1:20. Bajo estas condiciones, se observó una reducción de aproximadamente el $91 \%$ de los tensoactivos a los 120 min de tratamiento. El valor de concentración correspondiente $(0,45 \mathrm{mg} / \mathrm{L})$ se encontró por encima del límite máximo establecido por la norma de la EPA para descargas a un cuerpo de agua dulce, sin embargo, se encontró por debajo de $0,50 \mathrm{mg} / \mathrm{L}$ que es el límite establecido para aguas destinadas a consumo humano que requieren un tratamiento convencional [7]. En cuanto al proceso de desinfección, se logró la inactivación total de coliformes fecales a los 15 min de iniciada la reacción.

\section{Determinación de la relación molar $\mathrm{Fe}^{2+}$ :EDTA y valor de $\mathbf{p H}$ del proceso Fenton modificado que permitiera la mayor reducción de los contaminantes en estudio}

En la Figura 4 se presentan los resultados de la variación en el tiempo del porcentaje remanente de tensoactivos en la muestra compuesta, tratada mediante un proceso Fenton modificado con EDTA. El valor de $\mathrm{pH}$ de 6,41 correspondió al valor de $\mathrm{pH}$ del efluente sin que haya sido acondicionado para iniciar la reacción. Se presentan, además, los resultados agrupados de acuerdo con el valor del $\mathrm{pH}$ al inicio de cada tratamiento, como se aprecia en la Figura 4 (b), (c), (d) y (e). Al comparar el proceso Fenton convencional con el proceso Fenton modificado con EDTA, se apreció que la velocidad a la que ocurrió la reacción en el segundo proceso fue mayor. Esto se pudo evidenciar por el hecho de que en el proceso Fenton modificado con EDTA se 
alcanzaron porcentajes de remoción de tensoactivos entre 70 y $98 \%$ a los 15 min, en contraste con los porcentajes de remoción entre 52 y $71 \%$ obtenidos en el proceso Fenton convencional, para el mismo tiempo de tratamiento. Existen algunos factores que explican el hecho de que la rapidez a la que se produjo el proceso Fenton modificado con un agente quelante haya sido mayor que la del proceso convencional. Se ha evidenciado que al usar ácidos carboxílicos como agentes quelantes del hierro, se forman complejos capaces de generar especies ferrilo, (en las que el hierro presenta un estado de oxidación superior a 3), las cuales son agentes altamente oxidantes, por lo que contribuirían sinérgicamente con la acción de los 'OH para acelerar las reacciones de oxidación durante el proceso Fenton [9, 22].

Asimismo, se ha propuesto que el incremento en la velocidad de reacción en el proceso Fenton modificado se debe a que éste se produce por catálisis homogénea, en la cual, el catalizador es el complejo EDTA-Fe ${ }^{2+}$ y la velocidad de reacción depende de la concentración de este compuesto [29]; este último punto ha sido estudiado por Isch [30], en cuya investigación se degradó el agente tensoactivo lauril sulfato de sodio (LSS), por medio de un proceso Fenton modificado con EDTA y se confirmó la acción catalítica del complejo EDTA$\mathrm{Fe}^{2+}$. El análisis estadístico de los resultados reveló que únicamente el tiempo y el valor del $\mathrm{pH}$ al que se realizó el tratamiento tuvieron un efecto estadísticamente significativo durante el proceso, es decir, la relación molar $\mathrm{Fe}^{2+}$ :EDTA, en el rango utilizado en esta etapa, no tuvo dicho efecto sobre el proceso. En estudios previos se ha propuesto que el hecho de trabajar con un exceso de EDTA frente al hierro garantizaría el acomplejamiento completo del metal y permitiría así que la reacción ocurriese
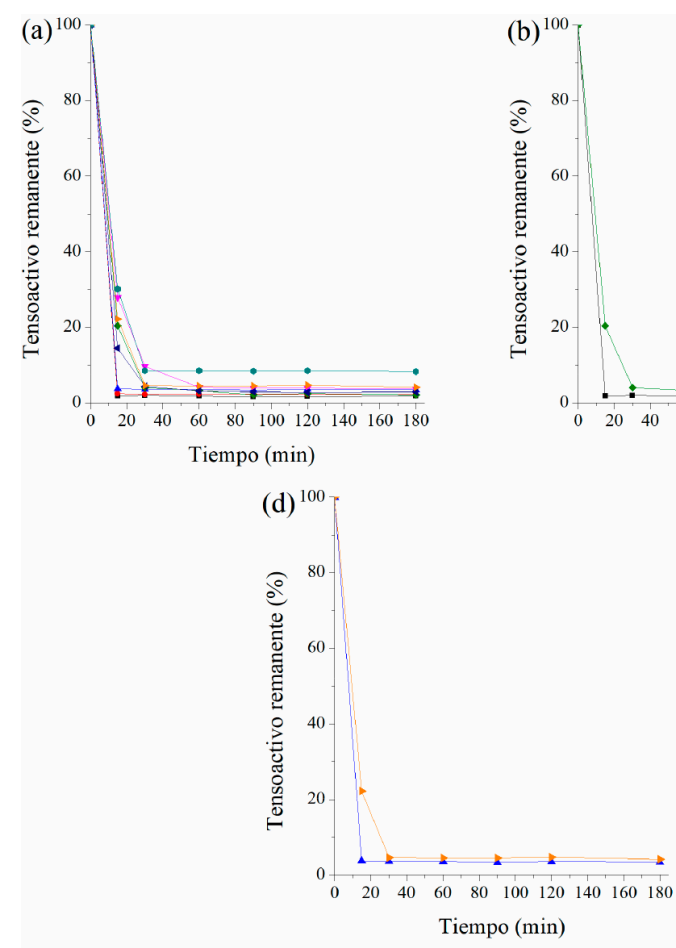

- $\mathrm{Fe}^{2+}: \mathrm{EDTA}=1: 1 ; \mathrm{pH}=8,00$

- $\mathrm{Fe}^{2+}: \mathrm{EDTA}=1: 1 ; \mathrm{pH}=6,41$

$-\mathrm{Fe}^{2+}: \mathrm{EDTA}=4: 5 ; \mathrm{pH}=8,00$
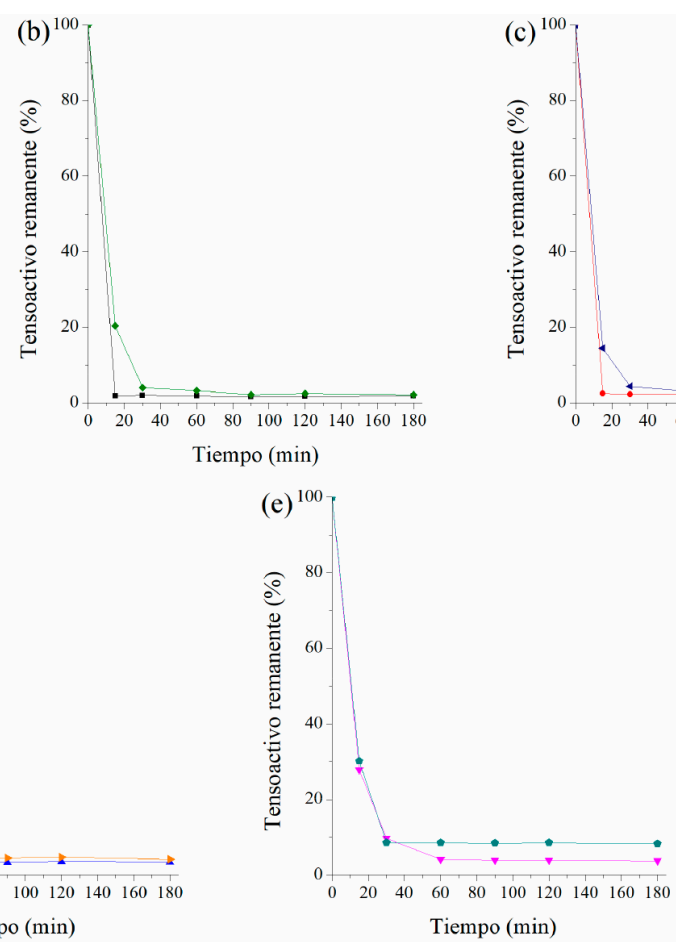

$\Delta \mathrm{Fe}^{2+}: \operatorname{EDTA}=1: 1 ; \mathrm{pH}=6,00$

$\nabla \mathrm{Fe}^{2+}: \mathrm{EDTA}=1: 1 ; \mathrm{pH}=4,50$ $\mathrm{Fe}^{2+}: \mathrm{EDTA}=4: 5 ; \mathrm{pH}=6,00 \bigcirc \mathrm{Fe}^{2+}: \mathrm{EDTA}=4: 5 ; \mathrm{pH}=4,50$

Figura 4. Variación del porcentaje remanente de tensoactivos (SAAM) en el efluente de la PTAR de Ubillús, tratado mediante Fenton modificado con EDTA con diferentes relaciones molares $\mathrm{Fe}^{2+}$ :EDTA y diferentes valores de $\mathrm{pH}$ : (a) todos los tratamientos; (b) tratamientos a $\mathrm{pH}=$ 8,00 ; (c) tratamientos a $\mathrm{pH}=6,41$; (d) tratamientos a $\mathrm{pH}=6,00$; (e) tratamientos a $\mathrm{pH}=4,50$ $\left(\left[\mathrm{H}_{2} \mathrm{O}_{2}\right]=124,4 \mathrm{mM} ; \mathrm{Fe}^{2+}: \mathrm{H}_{2} \mathrm{O}_{2}=1: 20\right.$; agitación = $\left.130 \mathrm{rpm} ;[\mathrm{SAAM}]_{0}=5,20 \mathrm{mg} / \mathrm{L}\right)$. 
en condiciones óptimas [12]. Sin embargo, los resultados obtenidos en el presente experimento mostraron un comportamiento opuesto al propuesto en la investigación anteriormente mencionada, ya que al trabajar con un exceso de EDTA respecto al hierro, dado por la relación molar $\mathrm{Fe}^{2+}$ :EDTA de 4:5, los porcentajes de remoción de tensoactivos fueron menores que los obtenidos al trabajar con una relación equimolar, es decir, $\mathrm{Fe}^{2+}$ :EDTA de 1:1.

La aparente desventaja de utilizar un exceso de quelante, se debió, probablemente, a que el complejo $\mathrm{Fe}^{2+}$ :EDTA se formó únicamente mediante una relación equimolar entre las dos sustancias [31] y, por tanto, las moléculas de quelante excedentes, que no formaron parte del complejo, pudieron actuar como scavengers de los ${ }^{\circ} \mathrm{OH}$, es decir, las moléculas de EDTA libres, al ser oxidables, compiten con los demás contaminantes presentes en el agua, lo cual afecta negativamente al rendimiento del proceso Fenton. No obstante, al comparar los casos en los que se usó una relación equimolar de $\mathrm{Fe}^{2+}$ :EDTA con los casos en los que se utilizó un exceso de quelante, se apreció que, para un mismo valor de $\mathrm{pH}$, a medida que avanzó la reacción los porcentajes de remoción de tensoactivos fueron similares. Con estos resultados se ratificó el hecho de que la relación molar $\mathrm{Fe}^{2+}$ :EDTA utilizada no tuvo efectos contrastantes en el proceso.

Como se mencionó anteriormente, el valor del pH influyó significativamente en el proceso con Fenton modificado. Se apreció que a medida que se incrementó el pH, la remoción de tensoactivos también aumentó. Éste comportamiento guardó una estrecha relación con la reactividad de las especies formadas por $\mathrm{el}^{2+}$, ya que un incremento en el valor del $\mathrm{pH}$ favorece la formación de especies como el complejo $\left[\mathrm{Fe}\left(\mathrm{H}_{2} \mathrm{O}\right)_{4}(\mathrm{OH})_{2}\right]$, cuya reactividad frente al $\mathrm{H}_{2} \mathrm{O}_{2}$ para producir ' $\mathrm{OH}$ es cerca de diez veces mayor que la del $\mathrm{Fe}^{2+}[9,32]$. El hecho de que el complejo EDTA- $\mathrm{Fe}^{2+}$ sea muy estable en un amplio rango de valores de $\mathrm{pH}$, probablemente, permitió que en el proceso Fenton modificado se puedan formar estas especies de $\mathrm{Fe}^{2+}$ de mayor reactividad, lo cual favoreció los procesos de oxidación de los contaminantes presentes en el agua.

Nuevamente, en todos los ensayos de esta etapa, se logró inactivación total de coliformes fecales, esto supuso una ventaja adicional del proceso Fenton modificado con EDTA, pues debido a la elevada reactividad de las especies que se generaron, permitió llevar a cabo el proceso de desinfección incluso en rangos de $\mathrm{pH}$ considerados adecuados para el crecimiento de $E$. coli $[2,11]$.

Si bien los mayores porcentajes de remoción de tensoactivos se alcanzaron en el tratamiento llevado a cabo a un valor de $\mathrm{pH}$ de 8,0 y con una relación molar $\mathrm{Fe}^{2+}$ :EDTA de 1:1, son comparables con los obtenidos en los procesos en los que se trabajó a 6,41 (que corresponde al $\mathrm{pH}$ del efluente) y 6,$0 ;$ con la misma relación molar. A pesar de las variaciones significativas, el trabajar directamente en el efluente sin que se acondicione el valor del $\mathrm{pH}$, supone el ahorro de tiempo y reactivos que se invertirían en este procedimiento; adicionalmente, en esta etapa, se ha probado la eficacia del tratamiento mediante Fenton modificado con EDTA en el rango de $\mathrm{pH}$ entre 6,0 y 8,0 ; valores que estuvieron dentro del rango establecido por las normas de la EPA y, además, estuvieron dentro de los límites de control establecidos en la etapa de caracterización, por lo que, prácticamente, se ha corroborado la eficacia del proceso en el rango de valores de $\mathrm{pH}$ que potencialmente podría presentar el efluente [8]. Por las razones expuestas anteriormente, se seleccionaron como las mejores condiciones de Fenton modificado con EDTA a la relación molar $\mathrm{Fe}^{2+}$ :EDTA de 1:1 y el valor de $\mathrm{pH}$ del efluente 6,41. Bajo estas condiciones se logró una remoción de aproximadamente el $97 \%$ de tensoactivos e inactivación total de coliformes fecales en un tiempo de $15 \mathrm{~min}$. La concentración correspondiente de tensoactivos fue de $0,13 \mathrm{mg} / \mathrm{L}$, valor que estuvo por debajo del límite máximo establecido por la norma de descarga a un cuerpo de agua dulce de la EPA [7].

\section{Evaluación de la remoción global de los contaminantes en el efluente, a través de la DQO, bajo las mejores condiciones de Fenton modificado con EDTA}

En la Tabla 2 se presentan los resultados obtenidos posteriores al tratamiento de una nueva muestra compuesta bajo las mejores condiciones de Fenton modificado con EDTA.

En primer lugar, se observó que los valores iniciales de concentración de tensoactivos, coliformes fecales y $\mathrm{pH}$, se encontraron dentro de los límites de control establecidos en la etapa de caracterización, 
Tabla 2. Variación de la carga contaminante en el efluente de la PTAR de Ubillús, tratado mediante Fenton modificado con EDTA bajo las mejores condiciones definidas para el proceso $\left(\left[\mathrm{H}_{2} \mathrm{O}_{2}\right]=124,4 \mathrm{mM}\right.$; EDTA: $\mathrm{Fe}^{2+}: \mathrm{H}_{2} \mathrm{O}_{2}=1: 1: 20 ; \mathrm{t}=15 \mathrm{~min}$; $\mathrm{pH}=6,97)$.

\begin{tabular}{|l|r|r|}
\hline \multicolumn{1}{|c|}{ Parámetro } & \multicolumn{1}{c|}{$\begin{array}{c}\text { Valor } \\
\text { inicial }\end{array}$} & $\begin{array}{c}\text { Valor } \\
\text { final }\end{array}$ \\
\hline DQO (mg/L) & 980,00 & 64,00 \\
\hline Tensoactivos (mg/L) & 6,12 & 0,19 \\
\hline Coliformes fecales (UFC/mL) & 71000,00 & 0,00 \\
\hline
\end{tabular}

hecho que corroboró el criterio de poder tomar la muestra compuesta en cualquier día de operación normal de la planta. Por otro lado, se apreció que los porcentajes de remoción de los contaminantes (97\% para tensoactivos y $100 \%$ para coliformes fecales) fueron semejantes a los que se obtuvieron al tratar la anterior muestra compuesta bajo las condiciones de Fenton modificado con EDTA previamente establecidas.

A través de la DQO, se cuantificó la materia orgánica presente en el agua que es susceptible de oxidarse por reacciones químicas, lo que incluyó las sustancias bio y no biodegradables [2]. De este modo, la medición de la DQO permitió evaluar de manera global la remoción de los contaminantes para el proceso en estudio, pues del mismo modo en que la remoción total de coliformes fecales no garantizó la remoción total de tensoactivos, los porcentajes de remoción de tensoactivos alcanzados no garantizarían la remoción de otros contaminantes presentes en el agua residual.

El porcentaje de remoción de la DQO fue de aproximadamente $93 \%$, a partir de este resultado podría asumirse que el tratamiento propuesto permitió remover eficazmente coliformes fecales, tensoactivos y otros contaminantes presentes en el efluente de la PTAR de Ubillús.

\section{CONCLUSIONES}

Las concentraciones de tensoactivos y coliformes fecales en el efluente de la PTAR de Ubillús, medidas por un período de 8 semanas, excedieron el límite máximo establecido por la norma de descarga a un cuerpo de agua dulce de la EPA, mientras que los valores de $\mathrm{pH}$ se encontraron dentro del rango determinado por dicha norma.

Las mejores condiciones determinadas para reducir tensoactivos y coliformes fecales mediante un proceso Fenton convencional fueron el uso de una concentración de $\mathrm{H}_{2} \mathrm{O}_{2}$ de $124,4 \mathrm{mM}$; relación molar $\mathrm{Fe}^{2+}: \mathrm{H}_{2} \mathrm{O}_{2}$ de 1:20 y agitación continua de $130 \mathrm{rpm}$. Bajo estas condiciones, a partir de una muestra con concentración inicial de tensoactivos y coliformes fecales de 5,20 mg/L y 66000 UFC/ $\mathrm{mL}$, respectivamente, se alcanzó inactivación total de coliformes fecales a los 15 min y una concentración de tensoactivos de $0,45 \mathrm{mg} / \mathrm{L}$ a los 120 min de tratamiento.

Las mejores condiciones establecidas para reducir tensoactivos y coliformes fecales mediante un proceso Fenton modificado con EDTA fueron el uso de una concentración de $\mathrm{H}_{2} \mathrm{O}_{2}$ de $124,4 \mathrm{mM}$; relación molar EDTA: $\mathrm{Fe}^{2+}: \mathrm{H}_{2} \mathrm{O}_{2}$ de 1:1:20, agitación continua de $130 \mathrm{rpm}$ y $\mathrm{pH}$ del efluente $(6,41)$. Bajo estas condiciones, a partir de una muestra con concentración inicial de tensoactivos y coliformes fecales de $6,12 \mathrm{mg} / \mathrm{L}$ y $71000 \mathrm{UFC} /$ $\mathrm{mL}$, respectivamente, se alcanzó inactivación total de coliformes fecales y una concentración de tensoactivos de $0,19 \mathrm{mg} / \mathrm{L}$ a los $15 \mathrm{~min}$ de tratamiento.

La aplicación del tratamiento Fenton modificado con EDTA bajo las mejores condiciones establecidas, permitió alcanzar un valor de $64 \mathrm{mg} / \mathrm{L}$ de DQO en un tiempo de $15 \mathrm{~min}$, a partir de una carga inicial de DQO de $980 \mathrm{mg} / \mathrm{L}$.

Los valores de las concentraciones de tensoactivos y coliformes fecales, obtenidos posteriormente al tratamiento con las mejores condiciones establecidas para el proceso Fenton convencional y Fenton modificado con EDTA, estuvieron por debajo de los límites máximos respectivos establecidos por las normas de la EPA, lo cual supone una ventaja frente a los tratamientos convencionales.

Las limitaciones del proceso Fenton modificado radican en el proceso de recuperación del hierro, pues el complejo EDTA-Fe ${ }^{2+}$ presenta una gran estabilidad. No obstante, existen otros procesos, tales como la nanofiltración, que pueden ser aplicados para recuperar $\mathrm{y}$, posteriormente, reutilizar el complejo EDTA- $\mathrm{Fe}^{2}$. 
La eficacia del proceso Fenton modificado con EDTA podría ser aprovechada, potencialmente, para descontaminar aguas que presenten problemas similares a los del efluente en estudio.

\section{AGRADECIMIENTOS}

Los autores agradecen a la Escuela Politécnica Nacional y al Departamento de Ciencias Nucleares por el financiamiento del proyecto interno "Estudio de un proceso Fenton modificado con agentes quelantes para el tratamiento complementario de aguas residuales contaminadas con tensoactivos y coliformes, en dos parroquias del Distrito Metropolitano de Quito”.

\section{REFERENCIAS}

[1] World Water Assessment Programme. "The United Nations World Water Development Report 4: Managing Water under Uncertainty and Risk". UNESCO. París, Francia. 2012.

[2] Metcalf \& Eddy. "Ingeniería de aguas residuales. Tratamiento, vertido y reutilización". McGrawHill. Madrid, España. 2000.

[3] J. Sanz, J. Lombraña, A. De Luis, and F. Varona. "UV/ $/ \mathrm{H}_{2} \mathrm{O}_{2}$ chemical oxidation for high loaded effluents: A degradation kinetic study of las surfactant wastewaters". Environmental Technology. Vol. 24 No 7, pp. 903-911. 2003. DOI: 10.1080/09593330309385627.

[4] N. Ashbolt. "Microbial contamination of drinking water and disease outcomes in developing regions". Toxicology. Vol. 198 No 1-3, pp. 229-238. 2004. DOI: 10.1016/j. tox.2004.01.030.

[5] M. Scott and M. Jones M. "The biodegradation of surfactants in the environment". Biochimica et Biophysica Acta (BBA) - Biomembranes. Vol. $1508 \mathrm{~N}^{\circ} 1-2$, pp. 235-251. 2000. DOI: 10.1016/S0304-4157(00)00013-7.

[6] H. Huang, Q. Wu, X. Tang, R. Jiang, and $\mathrm{H}$. Hu. "Formation of haloacetonitriles and haloacetamides and their precursors during chlorination of secondary effluents". Chemosphere. Vol. 144 No 1, pp. 297-303. 2016. DOI: 10.1016/j.chemosphere. 2015.08.082.

[7] Environmental Protection Agency. "Water Quality Criteria 1972 (The blue Book)". Office of Water Regulation and Standard. Washington D.C., Estados Unidos. 1972.
[8] Environmental ProtectionAgency. "Water Quality Criteria for Water 1986 (The gold Book)". Office of Water Regulation and Standards. Washington. D.C., Estados Unidos. 1986.

[9] J.J. Pignatello, E. Oliveros, and A. MacKay. "Advanced Oxidation Processes for Organic Contaminant Destruction Based on the Fenton Reaction and Related Chemistry". Critical Reviews in Environmental Science and Technology. Vol. $36 \mathrm{~N}^{\circ}$ 1, pp. 1-84. 2006. DOI: $10.1080 / 10643380500326564$.

[10] S. Chitra, K. Paramasivan, A. Shanmugamani, S. Rao, and P. Biplob. "Advanced Oxidation Process for the Surfactant Wastes". Journal of Chemical Engineering and Chemistry Research. Vol. 1 N $^{o}$ 3, pp. 167-173. 2014.

[11] A. Selvakumar, M. Tuccillo, S. Muthukrishnan, and A. Ray. "Use of Fenton's Reagent as a Desinfectant". Remediation Journal. Vol. 19 $\mathrm{N}^{\mathrm{o}}$ 2, pp. 135-142. 2009. DOI: 10.1002/ rem.20208.

[12] A. De Luca, R. Dantas, and S. Esplugas. "Assessments of iron chelates efficiency for photo-Fenton at neutral $\mathrm{pH}$ ". Water Research. Vol. $61 \mathrm{~N}^{\circ}$ 1, pp. 232-242. 2014. DOI: $10.1016 /$ j.watres.2014.05.033.

[13] N. Klamerth, S. Malato, A. Agüera, and A. Fernández-Alba. "Photo-Fenton modified photo-Fenton at neutral $\mathrm{pH}$ for the treatment of emerging contaminants in wastewater treatment plant effluents: A comparison". Water Research. Vol. 47 N 2 , pp. 833-840. 2013. DOI: 10.1016/j.watres.2012.11.008.

[14] K. Welch, T. Davis, and S. Aust. "Iron Autoxidation and Free Radical Generation: Effects of Buffers, Ligands, and Chelators". Archives of Biochemistry and Biophysics. Vol. $397 \mathrm{~N}^{\circ}$ 2, pp. 360-369. 2002. DOI:10.1006/abbi.2001.2694.

[15] Y. Tang, H. Liu, L. Zhou, H. Ren, H. Li, J. Zhang, G. Chen, and C. Qu. "Enhanced Fenton-like oxidation of hydroxypropyl guar gum catalyzed by EDTA-metal complexes in a wide $\mathrm{pH}$ range". Water Science and Technology, Vol. 79 № 9, pp. 1667-1674. 2019. DOI: $10.2166 /$ wst.2019.169.

[16] Y. Zhang, G. Luo, Q. Wang, Y. Zhang, and M. Zhou. "Kinetic study of the degradation of rhodamine B using a flow-through UV/ electro-Fenton process with the presence of ethylenediaminetetraacetic acid". 
Chemosphere, Vol. 240 N $^{\circ}$ 1, pp. 1-9. 2020. DOI:10.1016/j.chemosphere.2019.124929

[17] APHA, AWWA and WEF. "Standard methods for the examination of water and wastewater". American Public Health Association. Washington D. C., Estados Unidos. 2012.

[18] Minnesota Mining and Manufacturing Company. "Guía de interpretación. Placas para recuento de E. coli y Coliformes". 3M. Madrid, España. 2002.

[19] M. Kitis, C.D. Adams, and G.T. Daigger. "The effects of Fenton's reagent pretreatment on the biodegradability of nonionic surfactants". Water Research. Vol. 33 $\mathrm{N}^{\mathrm{o}}$ 11, pp. 2561-2568. 1999. DOI: 10.1016/ S0043-1354(98)00476-X.

[20] S. Lewis, A. Lynch, L. Bachas, S. Hampson, L. Ormsbee, and D. Bhattacharyya. "ChelateModified Fenton Reaction for the Degradation of Trichloroethylene in Aqueous and TwoPhase Systems". Environmental Engineering Science. Vol. 26 No 4, pp. 849-859. 2009. DOI: 10.1089/ees.2008.0277.

[21] C. Bouasla, M. Samar, and F. Ismail. "Degradation of methyl violet 6B dye by the Fenton process". Desalination. Vol. 254. No 1-3, pp. 35-41. 2010. DOI: 10.1016/j. desal.2009.12.017.

[22] P. Gogate \& A. Pandit. "A review of imperative technologies for wastewater treatment I: oxidation technologies at ambient conditions". Advances in Environmental Research. Vol. 8 No 3-4, pp. 501-555. 2004. DOI: 10.1016/ S1093-0191(03)00032-7.

[23] E. Neyens, and J. Baeyens. "A review of classic Fenton's peroxidation as an advanced oxidation technique". Journal of Hazardous Materials. Vol. 98 No 1-3, pp. 33-50. 2003. DOI: 10.1016/S0304-3894(02)00282-0.

[24] J. Kiwi, and V. Nadtochenko. "Evidence for the Mechanism of Photocatalytic Degradation of the Bacterial Wall Membrane at the $\mathrm{TiO}_{2}$ Interface by ATR-FTIR and Laser Kinetic Spectroscopy". Langmuir. Vol. 21 $\mathrm{N}^{\circ} 10$, pp. 4631-4641.2005. DOI: 10.1021/ la0469831.
[25] N. Klamerth, S. Malato, A. Agüera, A. FernándezAlba, and G. Mailhot. "Treatment of Municipal Wastewater Treatment Plant Effluents with Modified Photo-Fenton As a Tertiary Treatment for the Degradation of Micro Pollutants and Disinfection". Environmental Science \& Technology. Vol. $46 \mathrm{~N}^{\circ} 5$, pp. 2885-2892. 2012. DOI: $10.1021 / \mathrm{es} 204112 \mathrm{~d}$.

[26] T.G. Ellis, X. Huang, and S.K. Kaiser. "Linear alkybenzene sulfonate and alkyl sulfate biodegradation in laboratory and field activated sludge systems". WIT Transactions on Ecology and the Environment. Vol. 95 $\mathrm{N}^{\mathrm{o}}$ 1, pp. 551-560. DOI:10.2495/WP060541.

[27] J. Anotaia, M. Lu, and P. Chewpreecha. "Kinetics of aniline degradation by Fenton and electro-Fenton processes". Water Research. Vol. $40 \mathrm{~N}^{\circ}$ 9, pp. 1841-1847. 2006. DOI: 10.1016/j.watres.2006.02.033.

[28] A. Babuponnusami, and K. Muthukumar. "A review on Fenton and improvements to the Fenton process for wastewater treatment". Journal of Environmental Chemical Engineering. Vol. $2 \mathrm{~N}^{\mathrm{o}}$ 1, pp. 557-572. 2014. DOI: 10.1016/j.jece.2013.10.011.

[29] S. Bhaduri, and D. Mukesh. "Homogeneous Catalysis. Mechanisms and Industrial Applications". Wiley Interscience. New York, Estados Unidos. 2000.

[30] T. Isch. "Estudio de un sistema para el tratamiento de efluentes contaminados con compuestos surfactants por un método Fenton y Fenton modificado con un agente quelante EDTA". Escuela Politécnica Nacional. Quito, Ecuador. 2016.

[31] J. Bolobajev, M. Trapido, and A. Goi. "Improvement in iron activation ability of alachlor Fenton-like oxidation by ascorbic acid". Chemical Engineering Journal. Vol. $281 \mathrm{~N}^{\circ}$ 1, pp. 566-574. 2015. DOI: dx.doi.org/10.1016/j.cej.2015.06.115.

[32] C.F. Wells and M.A. Salam. "The effect of $\mathrm{pH}$ on the kinetics of the reaction of iron (II) with hydrogen peroxide in perchlorate media". Journal of the Chemical Society A: Inorganic, Physical, Theoretical. Vol. $1 \mathrm{~N}^{\circ}$ 1, pp. 24-29. 1968. DOI: $10.1039 / \mathrm{J} 19680000024$. 